\title{
Micosis fungoide hiperpigmentada de cara y pliegues en adultos: estudio prospectivo de una nueva variedad clínica
}

\author{
Hyperpigmented mycosis fungoides of the face and folds \\ in adults: a prospective study of a new clinical variety
}

\author{
María Antonieta Domínguez-Gómez, ${ }^{*}$ Karla Patricia Estrada-Ramírez, ${ }^{\ddagger}$ \\ Martha Alejandra Morales-Sánchez, ${ }^{\S}$ Fermín Jurado-Santa Cruz, " Gisela Navarrete-Franco," \\ José Alberto Ramos-Garibay, ${ }^{* *}$ Maribet González-González, ${ }^{* *}$ María Luisa Peralta-Pedrero ${ }^{\ddagger \ddagger}$
}

\section{RESUMEN}

La micosis fungoide es el linfoma cutáneo de células T reportada con mayor frecuencia a nivel mundial, cuenta con numerosas variantes, tanto desde el punto de vista clínico como histopatológico. La presentación en forma de manchas hiperpigmentadas es una variedad rara. En el Centro Dermatológico «Dr. Ladislao de la Pascua» de la Ciudad de México se ha detectado una manifestación especial de la misma, con afectación de cara y pliegues, la cual no ha sido descrita previamente. Objetivo: Comunicar las características clínicas e histopatológicas de estos casos, así como su tratamiento. Material y métodos: Se realizó un estudio de cohorte prospectivo de pacientes con diagnóstico clínico e histopatológico de micosis fungoide hiperpigmentada de cara y pliegues. Resultados: De 290 pacientes con diagnóstico de micosis fungoide estudiados en este centro dermatológico en un periodo de 12 años, 27 (9.3\%) presentaron lesiones hiperpigmentadas en cara y pliegues, 23 de ellos (85\%) pertenecían al sexo femenino, el grupo de edad más afectado se encontró entre los 46 y 56.3 años, con tiempo promedio de evolución de $12 \pm$ 91.88 meses (mínimo de 1 mes y máximo de 384 meses). La mediana de tiempo para el diagnóstico fue de 157 días $(15-2,155)$. Veinte pacientes

\section{ABSTRACT}

Mycosis fungoides (MF) is the most common cutaneous T-cell lymphoma (CTCL) worldwide, which has numerous variants, both from a clinical and histopathological point of view. The presentation in the form of hyperpigmented spots is a rare variety. At the "Dr. Ladislao de la Pascua" Dermatological Center a special manifestation of it has been detected, with affectation of the face and folds, which has not been previously described. Objective: Communicate the clinical and histopathological characteristics of these cases, as well as their treatment. Material and methods: A prospective cohort study of patients with a clinical and histopathological diagnosis of hyperpigmented mycosis fungoides of the face and folds was carried out. Results: Of 290 patients with a diagnosis of MF studied in this Dermatological Center over a period of 12 years, 27 patients (9.3\%) presented hyperpigmented lesions on the face and folds, 23 of them (85\%) belonged to the female sex, the oldest age group. Affected was between 46 and 56.3 years old, with an evolution time of 12 months \pm 91.88 (minimum of 1 month and maximum of 384 months). The median time to diagnosis was 157 days $(15-2,155)$. $74 \%$ (20) had head disease, 88\% (24) neck, 70\% (19) trunk, 55\% (15) upper extremities and

\footnotetext{
* Dermatóloga. Encargada del Servicio de Fototerapia.

₹ Dermatóloga Egresada.

$\S$ Dermatóloga. Maestra en Ciencias. Jefe de Enseñanza.

" Dermatólogo. Encargado de la Dirección.

I Encargada del Servicio de Dermatopatología.

** Dermatopatólogo.

\# Maestra en Ciencias. Adjunta del Curso de Dermatología.
}

Centro Dermatológico «Dr. Ladislao de la Pascua», SSCDMX.

Citar como: Domínguez-Gómez MA, Estrada-Ramírez KP, Morales-Sánchez MA, Jurado-Santa Cruz F, Navarrete-Franco G, Ramos-Garibay JA et al. Micosis fungoide hiperpigmentada de cara y pliegues en adultos: estudio prospectivo de una nueva variedad clínica. Rev Cent Dermatol Pascua. 2021; 30 (3): 131-138. https://dx.doi.org/10.35366/103826 
Domínguez-Gómez MA y cols. Micosis fungoide hiperpigmentada de cara y pliegues en adultos

(74\%) tuvieron afección de cabeza, $24(88 \%)$ en cuello, 19 (70\%) en tronco, $15(55 \%)$ en extremidades superiores y tres (11\%) en inferiores. Los resultados histopatológicos fueron reportados como concluyentes de MF en 14 casos (52\%), compatible en ocho (30\%) y en cinco se integró el diagnóstico por correlación clínico-histopatológica. En 22 pacientes $(81 \%)$ se indicó tratamiento con la aplicación de un inhibidor de calcineurina tópico, presentando remisión y despigmentación paulatina. En los demás se utilizaron diferentes medicamentos. La limitante de este estudio fue que, al considerarla una nueva variedad clínica, se requiere realizar estudios de inmunofenotipo y de genética. Conclusión: Consideramos que la micosis fungoide hiperpigmentada de cara y pliegues es una variante clínica no descrita previamente, por lo que proponemos que se incluya dentro de las variedades clínico-patológicas de linfoma cutáneo de células T.

Palabras clave: Micosis fungoide, hiperpigmentación de pliegues, linfoma cutaneo de células T.
$11 \%$ (3) lower. Histopathological results were reported as definitive MF in $52 \%$ (14) of the cases, compatible in $30 \%$ (8) and in 5 of the cases the diagnosis was made by clinical-histopathological correlation. Treatment with a topical calcineurin inhibitor was indicated in 22 patients (81\%) with remission and gradual depigmentation. Different medications were used in the others. The limitation of this study was that, as it is a new clinical variety, it is necessary to carry out immunophenotype and genetic studies. Conclusion: We consider that hyperpigmented mycosis fungoides of the face and folds is a clinical variant not previously described, so we propose that it be included within the clinic-pathological varieties of cutaneous T-cell lymphoma.

Keywords: Mycosis fungoides, hyperpigmentation of the folds, cutaneous T-cell lymphoma.

\section{INTRODUCCIÓN}

Los linfomas cutáneos primarios se definen como linfomas no-Hodgkin, los cuales se manifiestan en la piel sin evidencia de compromiso sistémico. El linfoma cutáneo de células T (LCCT) representa la mayoría de los linfomas cutáneos primarios en 75$80 \%$ de los casos, y el linfoma cutáneo de células B en $20-25 \% .{ }^{1}$ La micosis fungoide (MF) y el Síndrome de Sézary son las variedades clásicas del grupo de LCCT. Éstos forman parte de una familia de neoplasias primarias linfoides extranodales que se desarrollan de la transformación maligna de células T postímicas que invaden la piel. La MF se caracteriza por proliferación excesiva y aberrante de células T CD4 que se ubican en la piel, típicamente con fenotipo TH2. ${ }^{1-3}$ La MF es la entidad más común de LCCT; representa cerca de la mitad de todos los linfomas cutáneos primarios. ${ }^{4,5}$ La mayoría de los casos se desarrollan en personas mayores de 50 años. ${ }^{6}$

El diagnóstico y clasificación de los linfomas cutáneos primarios se basan en las características clínicas y en el resultado de los estudios histopatológicos, de inmunofenotipo y genéticos. La demostración de la presencia de un receptor clonal de células $\mathrm{T}$, o de los rearreglos genéticos de las inmunoglobulinas en la piel lesionada, o en sangre periférica, es de gran utilidad en casos específicos. Las características clínicas e histopatológicas son, en la mayoría de los casos, los factores decisivos y más importantes para iniciar el abordaje terapéutico. ${ }^{7}$

En los últimos 30 años la lista de diagnósticos diferenciales de MF ha ido en aumento, debido a la descripción de numerosas formas clínicas atípicas de MF que difieren de la presentación clínica clásica. Estas variantes pueden simular patologías inflamatorias de la piel, tanto a nivel clínico, como en la histopatología. ${ }^{6}$ Sin embargo, la clasificación por consenso de la Organización Mundial de la Salud y la Organización Europea para la Investigación y Tratamiento de Cáncer (WHOEORT) 2018, publicada en la cuarta edición del libro WHO Classification of Skin Tumours, sólo reconoce tres variantes diferentes, además de la forma clásica: MF foliculotrópica (MFF), reticulosis pagetoide y piel laxa granulomatosa, cada una con sus características clínico-patológicas, comportamiento clínico y/o pronóstico distintivos. ${ }^{1}$ Por su amplio espectro clínico-patológico, se considera a la MF como «la gran imitadora», por lo que se debe poner especial atención en los diagnósticos diferenciales. ${ }^{6}$

La MF hiperpigmentada es una variante clínica poco frecuente que ha sido comunicada como reporte y serie de casos. ${ }^{8-15}$ De forma similar a la variedad hipopigmentada, está subdiagnosticada en pacientes con fototipos cutáneos altos. Clínicamente se caracteriza por la presencia de manchas o placas hiperpigmentadas, de bordes más o menos definidos, con varios grados de atrofia y escama. Puede ser la única manifestación de MF, aunque algunos pacientes presentan lesiones concomitantes de MF clásica, u otras variantes, y puede simular gran variedad de entidades benignas. ${ }^{6,12}$

En el Centro Dermatológico Pascua se ha identificado una presentación clínica con hiperpigmentación de cara y pliegues no descrita antes. El objetivo de este trabajo es comunicar las características clínicas e histopatológicas de estos casos, así como su tratamiento. 


\section{MATERIAL Y MÉTODOS}

Población y diseño del estudio. En el Centro Dermatológico «Dr. Ladislao de la Pascua» de la Ciudad de México, del total de pacientes con diagnóstico de micosis fungoide estudiados durante el periodo comprendido entre los años 2007 y 2018 en la Unidad de Fototerapia se seleccionó a los pacientes con MF hiperpigmentada diseminada a cara y pliegues. Se recopilaron todos los datos clínicos, demográficos e iconográficos.

Estudio histopatológico. Los hallazgos del reporte histopatológico se clasificaron de acuerdo a lo establecido en el «Uso Apropiado de Criterios (AUC) en Dermatopatología para Enfermedades Linfoproliferativas de La Sociedad Americana de Dermatopatología», con la contribución de la Academia Americana de Dermatología y el Colegio Americano de Patólogos (Tabla 1). ${ }^{16,17}$

El estadiaje clínico se determinó utilizando el sistema TNMB de acuerdo a la Guía para el diagnóstico, tratamiento y seguimiento de linfomas cutáneos primarios publicada en 2018 por la Sociedad Europea de Medicina Oncológica (ESMO) para excluir el compromiso sistémico. $^{7}$

Análisis estadístico descriptivo. Se realizó utilizando medidas de tendencia central, de dispersión y porcentajes.

\section{RESULTADOS}

De los 290 pacientes con diagnóstico de MF, únicamente 33 presentaron lesiones hiperpigmentadas en cara y pliegues. De estos 33, se excluyeron seis por no cumplir con los criterios diagnósticos histopatológicos (tres con diagnóstico histopatológico de poiquilodermia, uno con imagen liquenoide y uno con melanosis).

Los 27 casos incluidos con MF hiperpigmentada de cara y pliegues, representan $9.3 \%$ del total de pacientes con diagnóstico de MF (Figura 1), 23 pertenecían al sexo femenino (85\%) y cuatro al masculino (15\%). La media de edad fue $46 \pm 10.30$ años. En relación con el tiempo de evolución, la media fue de $12 \pm 91.88$ meses, con un mínimo de un mes y máximo de 384 meses. La media de tiempo para el diagnóstico fue de 157 días $(15-2,155)$ (Tabla 2).

Doce pacientes (44\%) tuvieron afección a cabeza, cuello y tronco. Por segmentos corporales afectados, $20(74 \%)$ manifestaron afección a cabeza, 24 (89\%) a cuello, $19(70 \%)$ a tronco, $15(55 \%)$ a extremidades superiores y tres (11\%) a inferiores. Todos presentaron
Tabla 1: Uso apropiado de criterios para el diagnóstico

de micosis fungoide de la Sociedad Americana de

Dermatopatología con la contribución de la Academia Americana

de Dermatología y el Colegio Americano de Patólogos.

«Diagnóstico de» micosis fungoide:

- Presencia de casi todas las características histopatológicas típicas diagnósticas de micosis fungoide (linfocitos atípicos con núcleo hipercromático cerebriforme rodeado de halos claros, epidermotropismo de linfocitos solitarios o grupos de linfocitos atípicos en la ausencia de espongiosis, linfocitos epidérmicos más grandes que los dérmicos)

- Pérdida de uno o más marcadores importantes de células T (CD2, CD5 y/o CD7) con el infiltrado T neoplásico a lo largo de unión dermoepidérmica y/o en la epidermis

- Cerca del total de células neoplásicas expresan CD4 o CD8 (CD4 o CD8 con predominio significativo)

«Consistente con» micosis fungoide:

- Criterios de diagnóstico histopatológico presentes

- Linfocitos atípicos epidermotrópicos:

- Predominantemente inmunorreactivos para CD2, CD3, CD4, CD5 y CD7 (parcial)

- Predominantemente inmunorreactivos para CD4 o CD8

- Pérdida de uno o más marcadores de células T maduras (CD2, CD3, CD5, CD7)

«Sospecha de» 0 «Sugestivo de» micosis fungoide:

- Presencia de uno o más criterios de diagnóstico histopatológico típicos de micosis fungoide

- Linfocitos atípicos con núcleo hipercromático cerebriforme rodeado de halos claros

- Epidermotropismo de linfocitos solitarios o grupos de linfocitos atípicos en la ausencia de espongiosis

- Linfocitos epidérmicos más grandes que los dérmicos

- Distribución perivascular de los linfocitos atípicos

- Fibrosis papilar dérmica

- Características inmunofenotípicas normales: infiltrado de células T a lo largo de unión dermoepidérmica y/o en la epidermis que es inmunorreactivo a CD2, CD3, CD5 y CD7 (parcial o sin pérdida) con una proporción $\mathrm{CD} 4: \mathrm{CD} 8$ normal

«No diagnóstica de» micosis fungoide:

- Infiltrado de células T linfocitarias limitado/mínimo/escaso a lo largo de la unión dermoepidérmica y/o en la dermis superficial perivascular

- Ausencia de epidermotropismo o foliculotropismo

- Ausencia de linfocitos atípicos

- Ausencia de fibrosis papilar dérmica

- Características inmunofenotípicas normales: infiltrado de células T a lo largo de unión dermoepidérmica y/o en la epidermis que es inmunorreactivo a CD2, CD3, CD5 y CD7 (parcial o sin pérdida) con una proporción $\mathrm{CD} 4: \mathrm{CD} 8$ normal

Tomada de: Vidal $\mathrm{Cl}$ et al. ${ }^{16}$ 


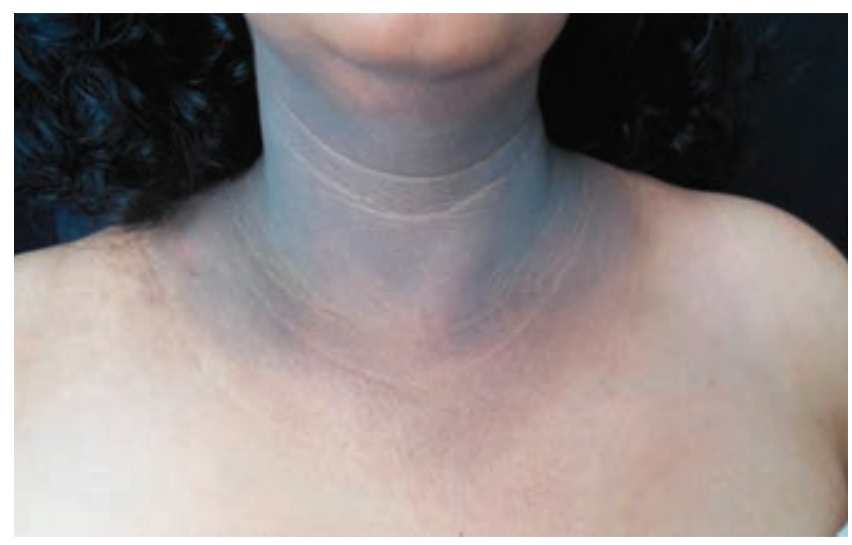

Figura 1: Manchas color marrón-grisáceo en cara anterior de cuello.

manchas hiperpigmentadas difusas. Cuatro también mostraron manchas hipopigmentadas, otros cuatro aspecto poiquilodérmico, dos eritema, uno pápulas foliculares y otro una placa eritematoescamosa.

Diecinueve pacientes $(70 \%)$ utilizaron tratamientos tópicos y de éstos, ocho (30\%) emplearon hidroquinona, siete $(26 \%)$ esteroides, cinco (19\%) emolientes y tres (11\%) «remedios caseros».

Doce pacientes (44\%) negaron haber sufrido enfermedades dentro de sus antecedentes patológicos, cuatro (15\%) padecían hipertensión arterial sistémica, tres $(11 \%)$ dislipidemia, uno diabetes mellitus tipo 2 y otro enfermedad autoinmune.

Como diagnóstico clínico inicial se sospechó de un proceso linfoproliferativo en 17 pacientes (63\%) y de éstos, en ocho (30\%) el diagnóstico fue de dermatitis cenicienta contra proceso linfoproliferativo (micosis fungoide). Dentro de los otros diagnósticos iniciales se consideraron: liquen plano pigmentado invertido, poiquilodermia atrófica vascular y parapsoriasis.

En cuanto a los hallazgos histopatológicos de los 27 pacientes, 24 (89\%) mostraron epidermotropismo, $22(81 \%)$ linfocitos perivasculares, $22(81 \%)$ caída del pigmento, $14(52 \%)$ hiperpigmentación de la capa basal, $15(56 \%)$ degeneración hidrópica de la capa basal, 11 (41\%) linfocitos atípicos, siete $(26 \%)$ microabscesos de Pautrier, tres (11\%) invasión folicular y tres $(11 \%)$ melanófagos (Figura 2 y Tabla 3).

De acuerdo con los criterios histopatológicos arriba mencionados, los resultados fueron reportados como compatibles con MF en ocho (30\%) de los casos, y concluyente en 14 (52\%). En cinco pacientes se integró el diagnóstico por correlación clínico-patológica, correspondiendo a la categoría "sugestivo de», de acuerdo con el AUC.
El número de biopsias requeridas para llegar al diagnóstico fue de una en 19 de los casos (70\%) y dos en ocho pacientes $(30 \%)$.

De acuerdo con el sistema TNMB, todos los casos se clasificaron como TIA.

Se indicó la aplicación de emolientes, protectores solares e inhibidores de calcineurina, este último fármaco en $22(81 \%)$ pacientes y PUVA en cabina vertical a siete (26\%). En cinco casos inhibidor de calcineurina tópico más PUVA en cabina vertical. A seis $(22 \%)$ aplicación de retinoides tópicos, a cinco (19\%) estreoides tópicos

Tabla 2: Características demográficas y clínico-histológicas de micosis fungoide hiperpigmentada de cara y pliegues de pacientes estudiados en el Centro Dermatológico «Dr. Ladislao de la Pascua».

\begin{tabular}{|c|c|}
\hline Características & $n(\%)$ \\
\hline Total & $27(100)$ \\
\hline \multicolumn{2}{|l|}{ Sexo } \\
\hline Femenino & $23(85)$ \\
\hline Masculino & $4(15)$ \\
\hline Edad (años), $M \pm D E$ & $46 \pm 10.3$ \\
\hline $\begin{array}{l}\text { Tiempo de evolución en meses, } \mathrm{M} \pm \mathrm{DE} \\
\text { (mín.-máx.) }\end{array}$ & $12 \pm 91.8(1-384)$ \\
\hline $\begin{array}{l}\text { Tiempo para el diagnóstico en días, } \mathrm{M} \pm \mathrm{DE} \\
\text { (mín.-máx.) }\end{array}$ & $157 \pm 476(15-2,155)$ \\
\hline \multicolumn{2}{|l|}{ Segmento corporal afectado } \\
\hline Cabeza & $20(74)$ \\
\hline Cuello & $24(89)$ \\
\hline Tronco & $19(70)$ \\
\hline Extremidades superiores & $15(56)$ \\
\hline Extremidades inferiores & $3(11)$ \\
\hline Cabeza, cuello y tronco & $12(44)$ \\
\hline \multicolumn{2}{|l|}{ Presentación clínica } \\
\hline $\begin{array}{l}\text { Lesiones de aspecto macular } \\
\text { hiperpigmentadas }\end{array}$ & $27(100)$ \\
\hline $\begin{array}{l}\text { Lesiones de aspecto macular } \\
\text { hipopigmentadas }\end{array}$ & $4(15)$ \\
\hline Aspecto poiquilodérmico & $4(15)$ \\
\hline Pápulas foliculares & $1(4)$ \\
\hline Eritema & $1(4)$ \\
\hline \multicolumn{2}{|l|}{ Hallazgos histopatológicos } \\
\hline Epidermotropismo & $24(89)$ \\
\hline Linfocitos perivasculares & $22(81)$ \\
\hline Caída del pigmento & $22(81)$ \\
\hline Hiperpigmentación de la capa basal & $14(52)$ \\
\hline Degeneración hidrópica de la capa basal & $15(56)$ \\
\hline Linfocitos atípicos & $11(41)$ \\
\hline Microabscesos de Pautrier & $7(26)$ \\
\hline Invasión folicular & $3(11)$ \\
\hline Melanófagos & $3(11)$ \\
\hline
\end{tabular}

$\mathrm{M}$ = media; $\mathrm{DE}$ = desviación estándar; mín. = mínimo; máx. = máximo. 

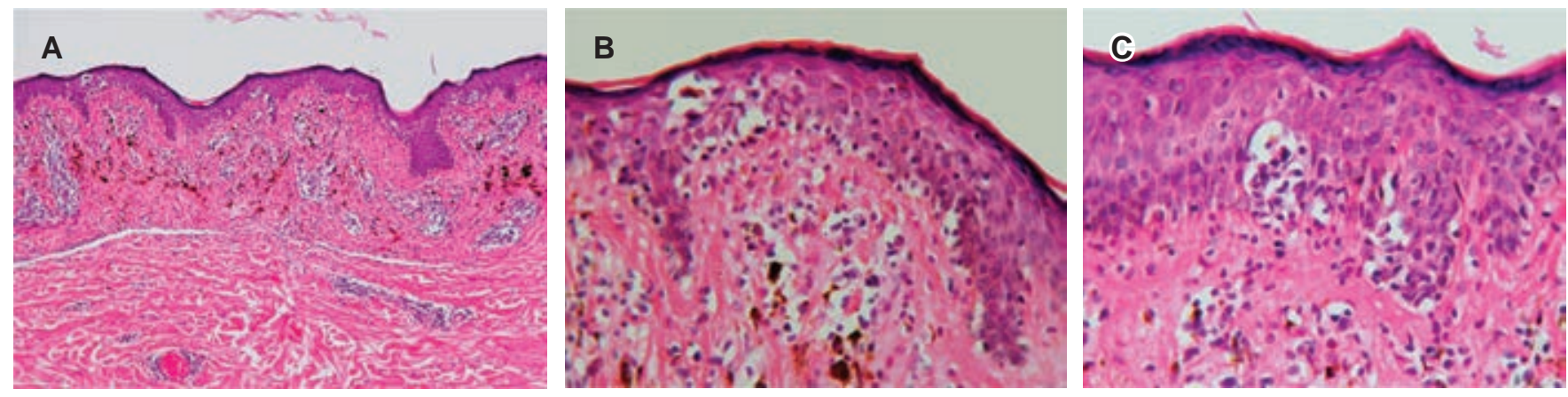

Figura 2: A) Epidermis con zonas de epidermotropismo. En la dermis, caída del pigmento e infiltrados moderados focales (H\&E 10x). B) A mayor aumento, epidermis con presencia de linfocitos atípicos y en dermis superficial, caída del pigmento y células del infiltrado (H\&E 40x). C) Presencia de microabscesos de Pautrier a nivel de epidermis (H\&E 40x).

Tabla 3: Hallazgos histológicos en 27 pacientes con micosis fungoide hiperpigmentada en cara y pliegues.

\begin{tabular}{lr} 
Hallazgos histológicos & $\mathrm{n}(\%)$ \\
\hline Características clásicas & \\
$\quad$ Epidermotropismo & $24(89)$ \\
Linfocitos perivasculares & $22(81)$ \\
Linfocitos atípicos & $11(41)$ \\
Microabscesos de Pautrier & $7(26)$ \\
Foliculotropismo & $3(11)$ \\
Hallazgos asociados con hiperpigmentación clínica & \\
Incontinencia del pigmento & $22(81)$ \\
Degeneración vacuolar difusa de los queratinocitos & $15(56)$ \\
basales & \\
Hiperpigmentación de los queratinocitos basales & $14(52)$ \\
Melanófagos & $3(11)$ \\
\hline
\end{tabular}

y a tres (11\%) PUVA-SOL. En el resto de los pacientes se observó remisión parcial de las lesiones hiperpigmentadas, con despigmentación paulatina (Figura 3). La recaída se presentó sólo en un caso.

\section{DISCUSIÓN}

En este artículo se comunica la descripción de las características clínico-histopatológicas de 27 pacientes con micosis fungoide hiperpigmentada de cara y pliegues, una variante clínica rara de linfoma cutáneo de células $\mathrm{T}$, no descrita antes en la literatura.

Todos fueron adultos, con predominio en mayores de 40 años (19 de 27 casos), similar a lo reportado a nivel internacional en $70 \%$ de pacientes con la variedad clásica de MF, en quienes se presenta en personas mayores de 50 años. ${ }^{6}$ Este rango de edad es similar a

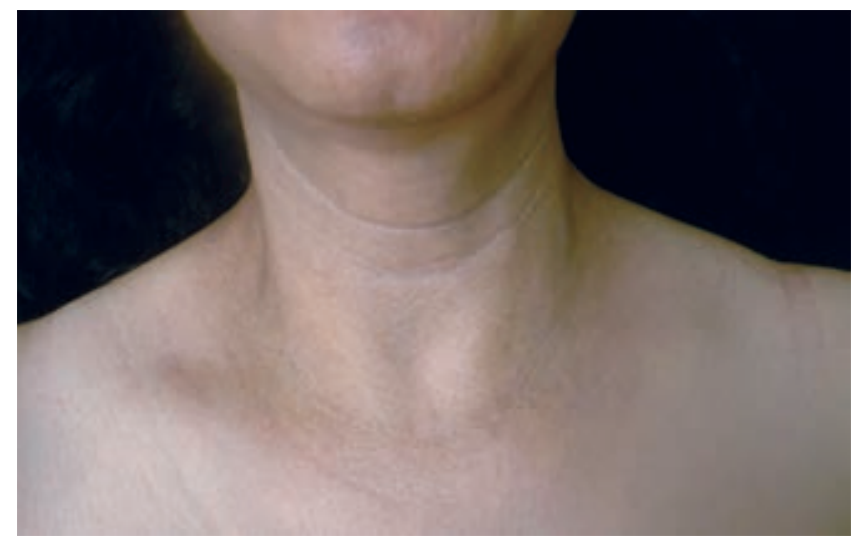

Figura 3: Mejoría clínica de la zona afectada, con despigmentación después del tratamiento.

la serie (ocho casos) de MF hiperpigmentada estudiada en un hospital de tercer nivel en Israel, en donde los autores reportan una media de edad de 43 años, y en la serie de casos de esta misma variedad analizada en un departamento de dermatología en Suiza (tres pacientes, de 42,45 y 66 años). ${ }^{12,18}$

Las lesiones hiperpigmentadas en nuestra cohorte estuvieron presentes exclusivamente en pacientes con fototipos cutáneos altos (> III), similar a los casos publicados con anterioridad. ${ }^{8-15}$ En 12 pacientes (44\%), las lesiones se ubicaron en cabeza, cuello y tronco.

Las lesiones hiperpigmentadas adquiridas tienen un espectro amplio de diagnósticos diferenciales, por lo que la MF hiperpigmentada puede simular gran variedad de entidades benignas: hiperpigmentación postinflamatoria, eritema pigmentado fijo, dermatitis de contacto, dermatitis cenicienta, amiloidosis cutánea, liquen plano pigmentado e hiperpigmentación macular eruptiva idiopática. ${ }^{6,12,19}$ 
Domínguez-Gómez MA y cols. Micosis fungoide hiperpigmentada de cara y pliegues en adultos

En el año 2016, se reportaron los casos de dos mujeres con hiperpigmentación de pliegues como manifestación inicial de MF, diagnósticados y tratados en la Unidad de Fototerapia del Centro Dermatológico Pascua, lo cual representó un importante desafio diagnóstico. En ambos casos la topografía afectada fue la cabeza, el cuello y el tronco; se manifestaron con manchas hiperpigmentadas mal definidas. Una de las pacientes señaló tener prurito y la otra cursó asintomática. Se les realizó estudio de inmunohistoquímica. Uno de los casos resultó con inmunofenotipo CD8+, y el otro fue CD4+. Se inició tratamiento con emolientes, protector solar e inhibidor de calcineurina cada 12 horas, (tratamiento no descrito previamente para MF con lesiones hiperpigmentadas). Con éste se logró la despigmentación paulatina y remisión total de las lesiones después de tres años de seguimiento. ${ }^{20}$

A partir de esa fecha, y después de estudiar estos casos, ante cuadros similares que acuden a esta unidad médica, se piensa ya en forma inicial que pueda tratarse de MF. Lo anterior explica por qué en el estudio referido en esta publicación se pensó desde el inicio en dicha patología en 17 (63\%) de los pacientes, y que de éstos, en ocho (30\%) el diagnóstico fuera de dermatitis cenicienta contra proceso linfoproliferativo. La media de tiempo para el diagnóstico fue de $157 \pm 476$ días, en el caso en el que hubo mayor retraso en el diagnóstico fue de 2,155 dias (70 meses), el diagnóstico inicial fue de dermatitis cenicienta, y fue tratada con hidroquinona durante ese tiempo.

En contraste con los casos reportados de MF variedad hiperpigmentada, en los que las lesiones se han descrito como manchas o placas hiperpigmentadas con escama en su superficie, ubicadas principalmente en tronco y extremidades, ${ }^{8-15}$ las lesiones en el grupo de pacientes estudiados en este centro se manifestaron como manchas hiperpigmentadas de color marrón o marrón violáceo, de límites difusos, localizadas en cara, cuello y zona $\mathrm{V}$ del escote.

En la serie de casos más grande de micosis fungoide hiperpigmentada reportada por Pimpinelli y colaboradores en el año 2005, ${ }^{21}$ con ocho casos, la presencia de discreta atrofia y escama en zonas de piel no fotoexpuestas fueron la clave para sospechar que se trataba de un proceso linfoproliferativo, ambas características de la MF clásica. ${ }^{21}$

En las dos últimas décadas se han propuesto diversos criterios citológicos e histopatológicos para identificar las categorías: «diagnóstico de», «consistente con» $\mathrm{y}$ «sugestivo de» MF, por lo que la Sociedad Americana de Dermatopatología con la contribución de la Academia
Americana de Dermatología y el Colegio Americano de Patólogos en el año 2018 desarrollaron el «Uso Apropiado de Criterios en dermatopatología para enfermedades linfoproliferativas». . $^{16,17,21}$ De acuerdo con esta clasificación, los resultados histopatológicos fueron reportados como compatibles con MF ocho $(30 \%)$ de los casos, concluyentes $14(52 \%)$ y en cinco pacientes se integró el diagnóstico por correlación clínico-histopatológica, cayendo en la categoría «sugestivo de». En el estudio histopatológico en un caso se reportó folículotropismo, y en los cinco casos arriba señalados, la presencia de linfocitos perivasculares. A nivel histopatológico, además de las características adicionales de la MF clásica, se han descrito la degeneración hidrópica de la capa basal simulando «dermatitis de la interfase» y la presencia de melanófagos en la mayoría de los casos. En relación con la inmunohistoquimica, la mayor parte de pacientes con MF hiperpigmentada muestran fenotipo CD8+. Se cree que estos linfocitos $T$ citotóxicos pueden afectar a los melanocitos o queratinocitos basales, resultando en cambios de la interfase e incontinencia del pigmento melánico, lo cual se manifiesta clínicamente como hiperpigmentación. ${ }^{12}$ En nuestro estudio, en 22 pacientes (81\%) se demostró la presencia de caída del pigmento, en 14 (52\%) hiperpigmentación de la capa basal, en 15 (56\%) degeneración hidrópica de la capa basal y en tres $(11 \%)$ melanófagos, hallazgos consistentes con la manifestación clínica.

En las dos series de MF hiperpigmentada de Israel y Suiza, ${ }^{12,18}$ los hallazgos histopatológicos en los tres casos de Israel fueron similares, con epidermotropismo de células individuales y discretos infiltrados perivasculares de pequeños linfocitos, así como marcada incontinencia del pigmento. En todos los casos de Suiza se observó dermatitis de interfase con melanófagos, además de epidermotropismo e infiltrado linfocitario dérmico.

El infiltrado inflamatorio crónico tiene un papel importante en el LCCT, con células estimuladas de forma paracrina. ${ }^{2,22}$ Las células T malignas tienen fenotipo de linfocitos de memoria CD4+, y presentan receptores que les guían a la piel, como el antígeno cutáneo linfocitario (ACL), así como el receptor CC de quimiocina 4 y 10 (CCR4 y CCR10). En la MF de bajo grado, las células T malignas también expresan el receptor $\mathrm{CC}$ de quimiocina 3 (CXCR3), que promueve su reclutamiento a la dermis y epidermis, donde son sintetizados sus ligandos correspondientes (CXCL9, CXCL10, CXCL11). Se ha reportado que el fenotipo de las células $T$ malignas corresponden a las células $T$ efectoras de memoria que residen en la piel; sin embargo, las características de las células $T$ malignas cambian durante la progresión 
de la enfermedad, por lo que parece haber un alto grado de heterogeneidad y plasticidad en las células malignas. ${ }^{22}$ En los estadios tempranos, las lesiones contienen pequeñas cantidades de poblaciones de células T malignas inmersas en un denso infiltrado de células inmunes reactivas. La progresión de la enfermedad está asociada con un aumento de marcadores de expresión Th2 como GATA-3 y citocinas (IL-4, IL-5 e IL-13) con la disminución de la expresión de factores Th1.22,23

Las células que infiltran la piel expresan el receptor de quimiocina 3 (CXCR3), el cual se une a tres ligandos diferentes: CXCL9 (monocina inducida por interferón gamma [IFN- $\gamma]$ ), CXCL10 (proteína-10 IFN inducible) y CXCL11 (célula T alfa quimiotáctico IFN inducible). Varios tipos de células expresan estas quimiocinas, incluyendo queratinocitos, fibroblastos dérmicos y células de Langerhans (LC). Las interacciones entre CXCL9, CXCL10 y CXCR3 son esenciales para el reclutamiento y acumulación de células tumorales en estadios tempranos. ${ }^{23}$ De esta manera, la buena respuesta al tratamiento con tacrolimus tópico al $0.1 \%$ probablemente se deba a que inhibe la proliferación de células $T$ y la producción de citocinas proinflamatorias como interleucinas IL-2, IL-3, IL-4, IL-12 y el factor de necrosis tumoral (TNF) e interferón IFN- $\gamma:{ }^{: 4}$ mecanismo con efecto antiinflamatorio, que favorece de manera secundaria la despigmentación, regulada por factores melanocíticos que son sintetizados de forma local por queratinocitos y fibroblastos, con regulación de tipo paracrina (las moléculas liberadas por una célula, actúan sólo sobre células vecinas) por medio de citocinas proinflamatorias producidas por los queratinocitos como IL1- $\alpha$ o TNF- $\alpha$, citocinas que estimulan a los fibroblastos, los cuales secretan factores estimuladores de los melanocitos como factor de células madre (SCF) y el factor de crecimiento de hepatocitos (HGF).

\section{CONCLUSIONES}

La variedad hiperpigmentada de la micosis fungoide es poco frecuente, y la que afecta pliegues lo es aún más. En este artículo se comunican casos de micosis fungoide hiperpigmentada de cara y pliegues, como una variante clínica no descrita previamente, y que por presentarse en zonas expuestas impacta en la calidad de vida de los pacientes, además de que el personal médico minimiza su importancia, o pueden ser diagnosticadas de manera errónea, y tratadas con despigmentantes, lo cual puede agravar el cuadro o retrasar el diagnóstico. Considerar esta variante clínica conducirá a un mejor manejo de los pacientes y se ten- drá la posibilidad de realizar un diagnóstico oportuno, y la remisión de las lesiones.

La limitante de esta investigación es que, al tratarse de una nueva variedad clínica de micosis fungoide, se requiere revisar en forma integral a cada paciente, solicitando estudios genéticos y de inmunofenotipo.

\section{REFERENCIAS}

1. Willemze R, Cerroni L, Kempf W, Berti E, Facchetti F, Swerdlow SH et al. The 2018 update of the WHO-EORTC classification for primary cutaneous lymphomas. Blood. 2019; 133: 1703-1714. doi: 10.1182/ blood-2018-11-881268.

2. Iqbal J, Amador C, McKeithan TW, Chan WC. Molecular and genomic landscape of peripheral T-cell lymphoma. Cancer Treat Res. 2019; 176: 31-68. doi: 10.1007/978-3-319-99716-2_2.

3. Shalabi D, Bistline A, Alpdogan O, Kartan S, Mishra A, Porcu P et al. Immune evasion and current immunotherapy strategies in mycosis fungoides (MF) and Sézary syndrome (SS). Chin Clin Oncol. 2019; 8: 11. doi: 10.21037/cco.2019.01.01.

4. KempfW,Zimmermann AK, Mitteldorf C. Cutaneous lymphomas-An update 2019. Hematol Oncol. 2019; 37 Suppl 1: 43-47. doi: 10.1002/hon.2584.

5. Willemze R, Jaffe ES, Burg G, Cerroni L, Berti E, Swerdlow SH et al. WHO-EORTC classification for cutaneous lymphomas. Blood. 2005; 105: 3768-3785. doi: 10.1182/blood-2004-09-3502.

6. Hodak E, Amitay-Laish I. Mycosis fungoides: a great imitator. Clin Dermatol. 2019; 37: 255-267. doi: 10.1016/j.clindermatol.2019.01.004.

7. Willemze R, Hodak E, Zinzani PL, Specht L, Ladetto M; ESMO Guidelines Committee. Primary cutaneous lymphomas: ESMO Clinical Practice Guidelines for diagnosis, treatment and follow-up. Ann Oncol. 2018; 29: iv30-iv40. doi: 10.1093/annonc/mdy133.

8. Puig L, Musulén E, Fernández-Figueras MT, Miralles J, Sitjas D, De Moragas JM. Mycosis fungoides associated with unusual epidermal hyperplasia. Clin Exp Dermatol. 1996; 21:61-64. Available in: http://www. ncbi.nlm.nih.gov/pubmed/8689775. Accessed November 16, 2019.

9. David M, Shanon A, Hazaz B, Sandbank M. Diffuse, progressive hyperpigmentation: an unusual skin manifestation of mycosis fungoides. J Am Acad Dermatol. 1987; 16: 257-260. doi: 10.1016/ s0190-9622(87)80076-2.

10. Erbil H, Sezer E, Koseoglu D, Filiz N, Kurumlu Z, Bülent Tastan $\mathrm{H}$ et al. Hyperpigmented mycosis fungoides: a case report. J Eur Acad Dermatol Venereol. 2007; 21: 982-983. doi: 10.1111/j.14683083.2006.02058.x.

11. Lee JS, Yun SJ, Lee JB, Kim SJ, Won YH, Lee SC. A case of hyperpigmented mycosis fungoides: a rare variant. J Eur Acad Dermatol Venereol. 2007; 21: 983-985. doi: 10.1111/j.14683083.2006.02060.x.

12. Pavlovsky L, Mimouni D, Amitay-Laish I, Feinmesser M, David M, Hodak E. Hyperpigmented mycosis fungoides: an unusual variant of cutaneous T-cell lymphoma with a frequent CD8+ phenotype. J Am Acad Dermatol. 2012; 67: 69-75. doi: 10.1016/j. jaad.2011.06.023.

13. Soughi M, Mernissi FZ. Hyperpigmented mycosis fungoides: a rare variant. Pan Afr Med J. 2013; 15: 13. doi: 10.11604/ pamj.2013.15.13.2789. 
14. Kazakov DV, Burg G, Kempf W. Clinicopathological spectrum of mycosis fungoides. J Eur Acad Dermatol Venereol. 2004; 18: 397-415. doi: 10.1111/j.1468-3083.2004.00937.x.

15. Lu YY, Wu CH, Lu CC, Hong CH. Hyperpigmentation as a peculiar presentation of mycosis fungoides. An Bras Dermatol. 2017; 92: $92-94$. doi: 10.1590/abd1806-484.

16. Vidal $\mathrm{Cl}$, Armbrect EA, Andea AA, Bohlke AK, Comfere NI, Hughes SR et al. Appropriate use criteria in dermatopathology: Initial recommendations from the American Society of Dermatopathology. J Cutan Pathol. 2018; 45: 563-580. doi: 10.1111/cup.13142.

17. Task Force/Committee Members, Vidal Cl, Armbrect EA, Andea $\mathrm{AA}$, Bohlke AK, Comfere $\mathrm{NI}$ et al. Appropriate use criteria in dermatopathology: Initial recommendations from the American Society of Dermatopathology. J Am Acad Dermatol. 2019; 80: 189207.e11. doi: 10.1016/j.jaad.2018.04.033.

18. Dummer R, Kamarashev J, Kempf W, Häffner AC, Hess-Schmid M, Burg G. Junctional CD8+ cutaneous lymphomas with nonaggressive clinical behavior: a CD8+ variant of mycosis fungoides? Arch Dermatol. 2002; 138: 199-203. doi: 10.1001/archderm.138.2.199.

19. Kumarasinghe SPW, Pandya A, Chandran V, Rodrigues M, Dlova NC, Kang HY et al. A global consensus statement on ashy dermatosis, erythema dyschromicum perstans, lichen planus pigmentosus, idiopathic eruptive macular pigmentation, and Riehl's melanosis. Int J Dermatol. 2019; 58: 263-272. doi: 10.1111/ijd.14189.

20. Domínguez-Gómez MA, Navarrete-Franco G, Gonell-Torres RL. Hiperpigmentación en pliegues como manifestación de micosis fungoide. Comunicación de dos casos. Rev Cent Dermatol Pascua. 2016; 25:19-23.

21. Pimpinelli N, Olsen EA, Santucci M, Vonderheid E, Haeffner AC, Stevens $S$ et al. Defining early mycosis fungoides. J Am Acad Dermatol. 2005; 53: 1053-1063. doi: 10.1016/j.jaad.2005.08.057.

22. Krejsgaard T, LindahI LM, Mongan NP, Wasik MA, Litvinov IV, Iversen L et al. Malignant inflammation in cutaneous T-cell lymphoma-a hostile takeover. Semin Immunopathol. 2017; 39: 269-282. doi: 10.1007/ s00281-016-0594-9.

23. Miyagaki T, Sugaya M. Immunological milieu in mycosis fungoides and Sézary syndrome. J Dermatol. 2014; 41: 11-18. doi: 10.1111/13468138.12305.

24. Cury Martins J, Martins C, Aoki V, Gois AF, Ishii HA, da Silva EM. Topical tacrolimus for atopic dermatitis. Cochrane Database Syst Rev. 2015; 2015: CD009864. doi: 10.1002/14651858.CD009864.pub2.

\section{Conflicto de intereses: Ninguno.}

\section{Correspondencia:}

María Antonieta Domínguez-Gómez

Dr. Vértiz Núm. 464, Esq. Eje 3 Sur,

Col. Buenos Aires, 06780,

Alcaldía Cuauhtémoc,

Ciudad de México, México.

Tel: 55 5634-9919

E-mail: dominguez035@yahoo.com.mx 